\title{
Gain Bounds for Multiple Model Switched Adaptive Control of General MIMO LTI Systems
}

\author{
Dominic Buchstaller and Mark French
}

\begin{abstract}
For the class of MIMO minimal LTI systems controlled by an estimation based multiple model switched adaptive controller (EMMSAC), bounds are obtained for the closed loop $l_{p}$ gain, $1 \leq p \leq \infty$, from the input and output disturbances to the internal signals.
\end{abstract}

\section{INTRODUCTION}

In standard approaches to multiple model switched adaptive control (MMSAC) e.g. in the sense of Morse [10], [11], Liberzon [9], etc. an LTI controller is constructed for every member of a "candidate" plant set such that the corresponding plant, controller pair is closed loop stable. Then for all candidate plants, observers are employed and the plant corresponding to the observer with the smallest output error is considered to be the best candidate. The algorithm then sequentially switches to the controller corresponding to the best candidate.

For non-zero input and output disturbances and in the presence of unmodeled dynamics, the analysis of the closed loop system becomes difficult. This is reflected by the fact that although this approach has been around since the early 1950s and although it functions well in practice, nevertheless it shares the lack of workable theoretical robustness results with classical adaptive approaches [3], [8], although see [5] for recent results in classical adaptive control based on the nonlinear robust stability theory of [7].

A different approach to MMSAC introduced by [4], [12], which we call EMMSAC, is to employ estimators instead of observers. An estimator observes the input and output signals of a process and explains this "observation" by input and output disturbances acting on plant models. At time $k \in \mathbb{N}$ the algorithm then switches to the controller which corresponding plant model's estimator is able to "explain" the observation with smallest disturbance.

This change of perspective allowed the construction of gain bounds in [4], [12] for two distinct plant models, in [6] for the class of dead beat stabilisable systems, and in [2] the further construction of gain function bounds invariant to the size of the candidate plant set was given. In this paper we generalise [6] and [2] to the class of all finite dimensional MIMO LTI systems. Such gain bounds are motivated by robust stability considerations [7] and leads to the MMSAC counterparts of the robust stability results of [5] for classical adaptive controllers.

We will also show that the EMMSAC approach naturally leads to nonlinear generalisations, and substantively different assumptions (of a convexity type) are required for the realisation of nonlinear estimation based schemes than those required for nonlinear MMSAC (which are of a structural type to allow the construction of observers).

\section{DEFINITIONS}

\section{A. Norms and signals}

Let $\mathcal{S}=\operatorname{map}\left(\mathbb{N}, \mathbb{R}^{h}\right), h \in \mathbb{N}$. For $a \in \mathcal{S}, 1 \leq r<\infty$ define the norms

$$
\|a\|_{r}:=\left(\sum_{0 \leq i<\infty}|a(i)|^{r}\right)^{1 / r},\|a\|_{\infty}:=\sup _{0 \leq i<\infty}|a(i)| .
$$

Let the truncation operator $\mathscr{T}_{k}: \mathcal{S} \rightarrow \mathcal{S}$ be defined by

$$
\left(\mathscr{T}_{k} a\right)(i)=\left\{\begin{array}{cc}
a(i), & 0 \leq i \leq k \\
0, & \text { otherwise }
\end{array}, k \in \mathbb{N}\right.
$$

and for $i, k \in \mathbb{N}$ let the restriction operator $\mathscr{R}_{i, k}: \mathcal{S} \rightarrow$ $\mathbb{R}^{h(i+1)}$ be defined by

$$
\mathscr{R}_{i, k} a:=(a(k-i), a(k-i+1), \ldots, a(k-1), a(k)) .
$$

An operator $O: \mathcal{S} \rightarrow \mathcal{S}$ is said to be to be causal if

$$
\mathscr{T}_{k} O \mathscr{T}_{k} v=\mathscr{T}_{k} O v, \forall k \in \mathbb{N}, v \in \mathcal{S} .
$$

Finally let

$$
\mathcal{V}=l_{r}, 1 \leq r \leq \infty, \mathcal{V}_{e}:=\left\{v \in \mathcal{S} \mid \forall k \in \mathbb{N}: \mathscr{T}_{k} v \in \mathcal{V}\right\}
$$

where $\mathcal{V}_{e} \supseteq \mathcal{V}$ is the extended space of possibly unbounded signals.

\section{B. Plant and controller}

For $m, o \in \mathbb{N}$ let $\mathcal{U}=\mathcal{V}^{m}, \mathcal{Y}=\mathcal{V}^{o}, \mathcal{W}=\mathcal{U} \times \mathcal{Y}$, $\mathcal{W}_{e}=\mathcal{U}_{e} \times \mathcal{Y}_{e}$. Given a plant $P: \mathcal{U}_{e} \rightarrow \mathcal{Y}_{e}$ and a controller $C: \mathcal{Y}_{e} \rightarrow \mathcal{U}_{e}$ the closed loop system $[P, C]$ under consideration in Figure 1 is defined via the following set of system equations:

$$
\begin{aligned}
& y_{1}=P u_{1}, u_{0}=u_{1}+u_{2}, y_{0}=y_{1}+y_{2}, \\
& u_{2}=C y_{2}
\end{aligned}
$$

where $w_{0}=\left(u_{0}, y_{0}\right)^{\top} \in \mathcal{W}$ represents the input and output disturbances, $w_{1}=\left(u_{1}, y_{1}\right)^{\top} \in \mathcal{W}_{e}$ represents the plant input and output and $w_{2}=\left(u_{2}, y_{2}\right)^{\top} \in \mathcal{W}_{e}$ represents the observed signal or observation. By abuse of notation we let $w_{0}(-k)=w_{1}(-k)=w_{2}(-k)=0, \forall k \in \mathbb{N} \backslash\{0\}$.

Let $\mathcal{P}, \mathcal{C}$ be (plant, controller) parameter sets parametrising the class of all causal, MIMO minimal LTI systems. For all $p \in \mathcal{P}, c \in \mathcal{C}$ define the plant and controller operators

$$
\begin{aligned}
& P_{p}: \mathcal{U}_{e} \rightarrow \mathcal{Y}_{e}, P_{p}(-k)=0, k \in \mathbb{N} \\
& C_{c}: \mathcal{Y}_{e} \rightarrow \mathcal{U}_{e}, C_{c}(-k)=0, k \in \mathbb{N}
\end{aligned}
$$




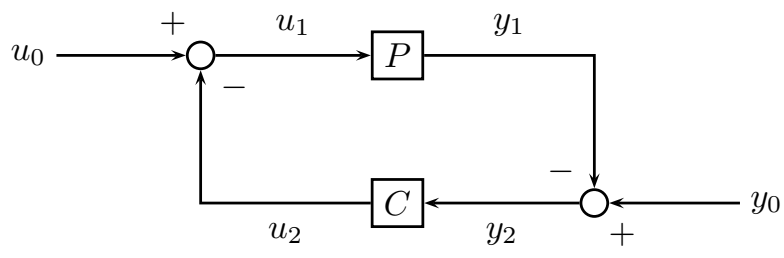

Fig. 1. The closed loop system $[P, C]$

and let

$$
\begin{aligned}
u_{2}^{c} & =C_{c} y_{2}^{c} \\
y_{1}^{p} & =P_{p} u_{1}^{p} \\
u_{0}^{p} & =u_{1}^{p}+u_{2} \\
y_{0}^{p} & =y_{1}^{p}+y_{2} .
\end{aligned}
$$

Define $\sigma(p), p \in \mathcal{P}$ to be the number of time steps the signal $w_{1}$ needs to be observed to uniquely determine the plant's initial condition. Respectively define $\sigma(c), c \in \mathcal{C}$ with respect to the signal $w_{2}^{c}=\left(u_{2}^{c}, y_{2}^{c}\right)^{\top}$.

\section{The closed loop operator}

Since we want to study the effect of input disturbances on the closed loop signals we introduce the closed-loop operator

$$
\Pi_{C / / P}: \mathcal{W} \rightarrow \mathcal{W}_{e}: w_{0} \mapsto w_{2}
$$

and define the gain

$$
\left\|\Pi_{C / / P}\right\|:=\sup _{w_{0} \in \mathcal{W} \backslash\{0\}} \frac{\left\|\Pi_{C / / P} w_{0}\right\|}{\left\|w_{0}\right\|}
$$

as well as the gain function

$$
g[P, C](r):=\sup _{w_{0} \in \mathcal{W},\left\|w_{0}\right\| \leq r}\left\|\Pi_{C / / P} w_{0}\right\| .
$$

We say that the closed loop $[P, C]$ is gain stable if $\left\|\Pi_{C / / P}\right\|<\infty$ and gain function stable if $g[P, C](r)<\infty$, $\forall r \geq 0$.

\section{The disturbance estimator}

One contribution of [6], [2] was the identification of necessary estimator properties for EMMSAC which completely separates the question of realisation of the disturbance estimation process from the stability analysis of the overall algorithm. We state these abstract assumptions (generalised from [2]) after introducing some necessary notation.

For $k \in \mathbb{N}, p \in \mathcal{P}$ define the estimation operator

$$
E: \mathcal{W}_{e} \rightarrow \operatorname{map}\left(\mathbb{N}, \operatorname{map}\left(\mathcal{P}, \operatorname{map}\left(\mathbb{N}, \mathbb{R}^{h}\right)\right)\right)
$$

by

$$
w_{2} \mapsto\left[k \mapsto\left(p \mapsto d_{p}[k]\right)\right]
$$

where $d_{p}[k]$ will represent the time series of disturbance estimates up to time $k$ corresponding to a plant $p \in \mathcal{P}$ denoted by

$$
d_{p}[k]: \mathbb{N} \rightarrow \operatorname{map}\left(\mathbb{N}, \mathbb{R}^{h}\right)
$$

and

$$
d_{p}[k]=\left(d_{p}[k](0), d_{p}[k](1), \ldots, d_{p}[k](k), 0, \cdots\right)
$$

where $h \in \mathbb{N}$ depends on $p \in \mathcal{P}$. We define the norm operator

$$
\begin{aligned}
N: \operatorname{map}\left(\mathbb{N}, \operatorname{map}\left(\mathcal{P}, \operatorname{map}\left(\mathbb{N}, \mathbb{R}^{h}\right)\right)\right) & \\
& \rightarrow \operatorname{map}\left(\mathbb{N}, \operatorname{map}\left(\mathcal{P}, \mathbb{R}^{+}\right)\right)
\end{aligned}
$$

by

$$
\left[k \mapsto\left(p \mapsto d_{p}[k]\right)\right] \mapsto\left[k \mapsto\left(p \mapsto\left\|d_{p}[k]\right\|\right)\right] .
$$

The operator $N E: \mathcal{W}_{e} \rightarrow \operatorname{map}\left(\mathbb{N}, \operatorname{map}\left(\mathcal{P}, \mathbb{R}^{+}\right)\right)$is called the disturbance estimator.

Define the set $W_{p}(i, k), k, i \in \mathbb{N}$ of weakly consistent disturbance signals at time $k$ of length $i$ to a plant $p \in \mathcal{P}$ and the observation $\left(u_{2}, y_{2}\right)^{\top}$ as follows:

$$
W_{p}(i, k):=\left\{\begin{aligned}
& v \in \mathbb{R}^{m(i+1)} \times \mathbb{R}^{n(i+1)} \\
& \exists\left(u_{0}^{p}, y_{0}^{p}\right)^{\top} \in \mathcal{W}_{e} \text { s.t. } \\
& \mathscr{R}_{i, k} P_{p}\left(u_{0}^{p}-u_{2}\right)=\mathscr{R}_{i, k}\left(y_{0}^{p}-y_{2}\right) \\
& v=\left(\mathscr{R}_{i, k} u_{0}^{p}, \mathscr{R}_{i, k} y_{0}^{p}\right)
\end{aligned}\right\}
$$

where we consider a vector $v \in \mathbb{R}^{m(i+1)} \times \mathbb{R}^{o(i+1)}$ to be weakly consistent over the interval $i$ with $\left(u_{2}, y_{2}\right)^{\top}$ and the plant $P_{p}$ if and only if $v \in W_{p}(i, k)$.

Let $p_{*}$ be the parameter corresponding to the "true" unknown plant $P:=P_{p_{*}} \in \mathcal{P}$.

Assumption 2.1: Let $\lambda \in \mathbb{R}$ be given.

1) (Causality): $E$ is causal.

2) (Minimality): There exists a $\mu>0$ such that for all $k \geq$ 0 , for $p \in \mathcal{P}$ and for all $\left(w_{0}, w_{1}, w_{2}\right) \in \mathcal{W} \times \mathcal{W}_{e} \times \mathcal{W}_{e}$ satisfying (2.1) for $P=P_{p}$

$$
N E\left(w_{2}\right)(k)(p)=\left\|\left[E\left(w_{2}\right)(k)\right](p)\right\| \leq \mu\left\|w_{0}\right\| .
$$

3) (Weak consistency): Let $0 \leq j \leq \lambda$. For all $p \in \mathcal{P}$ there exists

$$
\Phi_{j}: \operatorname{map}\left(\mathbb{N}, \mathbb{R}^{h}\right) \rightarrow \mathbb{R}^{m(j+1)} \times \mathbb{R}^{o(j+1)},
$$

such that for all $\left(w_{0}, w_{1}, w_{2}\right) \in \mathcal{W} \times \mathcal{W}_{e} \times \mathcal{W}_{e}$ satisfying (2.1) for $P=P_{p}$ and for all $k \in \mathbb{N}$,

$$
\Phi_{j} E\left(w_{2}\right)(k)(p) \in W_{p}(j, k)
$$

and

$$
\left\|\Phi_{j} E\left(w_{2}\right)(k)(p)\right\| \leq\left\|\mathscr{R}_{j, k} E\left(w_{2}\right)(k)(p)\right\| .
$$

4) (Monotonicity): For all $p \in \mathcal{P}$, for all $k, l \in \mathbb{N}$ with $0 \leq k \leq l$ and for all $\left(w_{0}, w_{1}, w_{2}\right) \in \mathcal{W} \times \mathcal{W}_{e} \times \mathcal{W}_{e}$ satisfying (2.1) for $P=P_{p}$ there holds

$$
\left\|E\left(w_{2}\right)(k)(p)\right\| \leq\left\|\mathscr{T}_{k} E\left(w_{2}\right)(l)(p)\right\| .
$$

We will now give two examples of estimators (from [6]) and show that they fullfill the given assumptions. It is important to note the the implementation of the EMMSAC controller requires a realisation of the operator $N E$ and it is only the analysis that requires the factorisation into the operators $N, E$. This important point is illustrated by our first example estimator (estimator A), which we give next. The direct formulation of estimator $\mathrm{A}$ is via the factorisation $N, E$. However, it is known in the $l_{2}$ setting that this deterministic least squares estimator $N E$ can be realised by a 
process of determining the residuals in a Kalman filter bank, see [4], [13].

For $p \in \mathcal{P}, k \in \mathbb{N}$ let estimator A with $h=\infty$ in equation (2.9) be given by:

$$
E_{A}\left(w_{2}\right)(k)(p)=\underset{x \in W_{p}(k, k)}{\operatorname{argmin}}\|x\|
$$

where $W_{p}(k, k)$ is the set of all truncated disturbance signals consistent with the observation $\mathscr{T}_{k} w_{2}$ an the plant $P_{p}$ over the interval $[0, k], k \in \mathbb{N}$.

Lemma 2.2: Estimator A fulfils assumptions 2.1.

Proof Let $k \in \mathbb{N}$. 1. Causality: The disturbance estimate at time $k \in \mathbb{N}$ does not depend on future information $\left.w_{2}\right|_{(k, \infty)}$ and is therefore causal. 2. Minimality: Observe that for any $\left(w_{0}, w_{1}, w_{2}\right) \in \mathcal{W} \times \mathcal{W}_{e} \times \mathcal{W}_{e}$ satisfying (2.1) for $P=P_{p}$ and for $k \in \mathbb{N}$ we have $\mathscr{T}_{k} w_{0} \in W_{p}(k, k)$. Hence $\left\|E_{A}\left(w_{2}\right)(k)(p)\right\|=\left\|\mathscr{T}_{k} \operatorname{argmin}_{x \in W_{p}(k, k)}\right\| x\|\| \leq$ $\left\|\mathscr{T}_{k} w_{0}\right\| \leq\left\|w_{0}\right\|$ and hence $\mu=1$. 3. Weak consistency: Let $0 \leq j \leq \lambda, p \in \mathcal{P}$. Let $\Phi_{j}$ be defined by $\Phi_{j} x=\mathscr{R}_{j, k} x, x \in \mathcal{S}$, and therefore $\left\|\Phi_{j} E_{A}\left(w_{2}\right)(k)(p)\right\|=$ $\left\|\mathscr{R}_{j, k} E_{A}\left(w_{2}\right)(k)(p)\right\|$. We then have $\Phi_{j} E_{A}\left(w_{2}\right)(k)(p)=$ $\mathscr{R}_{j, k} E_{A}\left(w_{2}\right)(k)(p) \in \mathscr{R}_{j, k} W_{p}(k, k) \subseteq W_{p}(j, k)$. Monotonicity: Let $p \in \mathcal{P}$, let $k \leq l, k, l \in \mathbb{N}$ and suppose $\left(w_{0}, w_{1}, w_{2}\right) \in \mathcal{W} \times \mathcal{W}_{e} \times \mathcal{W}_{e}$ satisfy equations (2.1) for $P=P_{p}$. Observe that $\mathscr{T}_{k} E_{A}\left(w_{2}\right)(l)(p) \in W_{p}(k, k)$. Since $E_{A}\left(w_{2}\right)(k)(p)=\operatorname{argmin}_{x \in W_{p}(k, k)}\|x\|$ it follows that $\left\|E_{A}\left(w_{2}\right)(k)(p)\right\| \leq\left\|\mathscr{T}_{k} E_{A}\left(w_{2}\right)(l)(p)\right\|$ as required.

The second example estimator (estimator B) is motivated by the fact that by Assumption 2.1(3) we only require consistency over suitable finite intervals of length $j \in \mathbb{N}, 0 \leq$ $j \leq \lambda$, where $\lambda$ is fixed. This allows for the construction of a finite horizon estimator as follows.

Let $\lambda \in \mathbb{N}$. For $p \in \mathcal{P}, 0 \leq i \leq k, k, i \in \mathbb{N}$ let estimator B with $h=(m+o)(\lambda+1)$ in equation (2.9) be given by:

$$
\begin{aligned}
E_{B}\left(w_{2}\right)(k)(p) & =d_{p}^{B}[k] \in \operatorname{map}\left(\mathbb{N}, \mathbb{R}^{h}\right) \\
d_{p}^{B}[k](i) & =\underset{x \in W_{p}(\lambda, i)}{\operatorname{argmin}}\|x\|,
\end{aligned}
$$

where $W_{p}(\lambda, i)$ is the set of all disturbance signals consistent with the observation $\mathscr{R}_{\lambda, i} w_{2}$ and the plant $P_{p}$ over the interval $[i-\lambda, i]$. Note that $d_{p}^{B}[k](j)=d_{p}^{B}[l](j)$ for $l \geq$ $k \geq j$.

Lemma 2.3: Estimator B fulfils assumptions 2.1.

Proof Let $k \in \mathbb{N}$. 1. Causality: $E_{B}$ is invariant to $\left.w_{2}\right|_{(k, \infty)}$. 2. Minimality: Observe that for any $\left(w_{0}, w_{1}, w_{2}\right) \in \mathcal{W} \times$ $\mathcal{W}_{e} \times \mathcal{W}_{e}$ satisfying (2.1) for $P=P_{p}$ and for $k \in \mathbb{N}$ we have $\mathscr{R}_{\lambda, i} w_{0} \in W_{p}(\lambda, i), 0 \leq i \leq k$. Hence $\left\|d_{p}^{B}[k](i)\right\|=$ $\operatorname{argmin}_{x \in W_{p}(\lambda, i)}\|x\| \leq\left\|\mathscr{R}_{\lambda, i} w_{0}\right\|, 0 \leq i \leq k, k \in \mathbb{N}$. Then we obtain

$$
\begin{aligned}
& \left\|E_{B}\left(w_{2}\right)(k)(p)\right\|=\left\|d_{p}^{B}[k](0), d_{p}^{B}[k](1), \ldots, d_{p}^{B}[k](k)\right\| \\
\leq & \left\|\mathscr{R}_{\lambda, 0} w_{0}, \mathscr{R}_{\lambda, 1} w_{0}, \cdots, \mathscr{R}_{\lambda, k} w_{0}\right\| \\
\leq & \| \begin{array}{cccc}
w_{0}(-\lambda), & w_{0}(1-\lambda), & \cdots, & w_{0}(k-\lambda) \\
w_{0}(1-\lambda), & w_{0}(2-\lambda), & \cdots, & w_{0}(k+1-\lambda) \\
\vdots & \vdots & \vdots & \vdots \\
w_{0}(0), & w_{0}(1), & \cdots, & w_{0}(k)
\end{array}= \\
= & (\lambda+1)^{1 / r}\left\|w_{0}\right\|=\mu\left\|w_{0}\right\|
\end{aligned}
$$

where the first inequality follows from the fact that \|\|$a\|\| b\|\|=,\|(a, b)\|$ holds in $l_{r}, 1 \leq r \leq \infty$. 3. Weak consistency: Let $0 \leq j \leq \lambda, p \in \mathcal{P}$. Let $\Phi_{j}$ be defined by $\Phi_{j} d_{p}^{B}[k]=\mathscr{R}_{j, \lambda} d_{p}^{B}[k](k)$. Since $\mathscr{R}_{j, \lambda} d_{p}^{B}[k](k) \subseteq \mathscr{R}_{j, k} d_{p}^{B}[k]$ there holds $\left\|\Phi_{j} E_{B}\left(w_{2}\right)(k)(p)\right\| \leq\left\|\mathscr{R}_{j, k} E_{B}\left(w_{2}\right)(k)(p)\right\|$. Also $\Phi_{j} d_{p}^{B}[k]=\mathscr{R}_{j, \lambda} d_{p}^{B}[k](k) \in W_{p}(j, k)$. 4. Monotonicity: Let $p \in \mathcal{P}$, let $k \leq l, k, l \in \mathbb{N}$ and suppose $\left(w_{0}, w_{1}, w_{2}\right) \in \mathcal{W} \times \mathcal{W}_{e} \times \mathcal{W}_{e}$ satisfy (2.1) for $P=P_{p}$. Since $\mathscr{T}_{k} d_{p}^{B}[l]=d_{p}^{B}[k]$ it follows that $\left\|E_{p}^{B}\left(w_{2}\right)(k)(p)\right\|=$ $\left\|\mathscr{T}_{k} E_{p}^{B}\left(w_{2}\right)(l)(p)\right\| \leq\left\|E_{p}^{B}\left(w_{2}\right)(l)(p)\right\|$.

Observe that the use of a finite horizon estimator in estimator $\mathrm{B}$ is penalised with a $\mu>1$. However the computational complexity of estimator $\mathrm{B}$ is invariant to $k$ and only depends on the horizon $i \in \mathbb{N}$. In contrast, the computational complexity of estimator A grows unboundedly with $k$ (with the important known exception of the Kalman filter realisation which holds only in the $l_{2}$ setting). We further remark that the finite horizon estimation computation of estimator B is a standard optimisation problem with many possible implementations. For example in $l_{2}$ we can solve the least squares problem by calculating a suitable pseudo inverse; in $l_{\infty}$ we can solve the convex optimisation problem by linear programming. In the nonlinear setting the given assumptions remain valid, and note that under appropriate convexity assumptions, the nonlinear optimisation problem remains computationally tractable.

\section{E. Finite horizon behaviour of the closed loop $\left[P_{p}, C_{c}\right]$}

One crucial design step for any MMSAC algorithm is to assign stabilising controllers to all members of the candidate plant set. We will do this via the design procedure given by the map

$$
K: \mathcal{P} \rightarrow \mathcal{C}
$$

We now state some general requirements on the atomic closed loop systems $\left[P_{p}, C_{c}\right]$ and $\left[P_{p}, C_{K(p)}\right]$ :

Assumption 2.4: There exist functions

$$
\alpha, \beta: \mathcal{P} \times \mathcal{C} \times \mathbb{R} \times \mathbb{R} \rightarrow \mathbb{R}
$$

such that the following hold:

1) (Linear growth of $\left[P_{p}, C_{c}\right]$ ): Let $p \in \mathcal{P}, c \in \mathcal{C}$. Let $l_{1}, l_{1}, l_{2}, l_{3}, l_{4} \in \mathbb{N}, l_{1}<l_{2} \leq l_{3}<l_{4}$ and $I_{1}=\left[l_{1}, l_{2}\right), I_{2}=\left[l_{2}, l_{3}\right), I_{3}=\left[l_{3}, l_{4}\right)$. Suppose $w_{2}, w_{2}^{c}, w_{1}^{p} \in \mathcal{W}_{e}, w_{0}^{p} \in \mathcal{W}$ satisfy equations (2.5)(2.8) on $I_{1} \cup I_{2} \cup I_{3}$. 
Suppose $\left.w_{2}^{c}\right|_{I_{1}} \in\left\{0,\left.w_{2}\right|_{I_{1}}\right\},\left.w_{2}^{c}\right|_{I_{2} \cup I_{3}}=\left.w_{2}\right|_{I_{2} \cup I_{3}}$ where

$$
\left|I_{1}\right|=l_{2}-l_{1} \geq \max \{\sigma(p), \sigma(c)\} .
$$

Then:

$$
\begin{aligned}
\left\|\left.w_{2}\right|_{I_{3}}\right\| \leq & \alpha\left(p, c,\left|I_{2}\right|,\left|I_{3}\right|\right)\left\|\left.w_{2}\right|_{I_{1}}\right\| \\
& +\beta\left(p, c,\left|I_{2}\right|,\left|I_{3}\right|\right)\left\|\left.w_{0}^{p}\right|_{I_{1} \cup I_{2} \cup I_{3}}\right\| .
\end{aligned}
$$

2) (Stability of $\left[P_{p}, C_{K(p)}\right]$ ): Let $p \in \mathcal{P}$ and $x \in \mathbb{N}$. Then

$$
\alpha(p, K(p), a, x) \rightarrow 0 \text { as } a \rightarrow \infty
$$

and $\alpha$ is monotonic in $a$.

We remark that the above assumptions are in fact standard properties for minimal LTI systems $P_{p}, C_{c}$. Such plants and controllers satisfy Assumption 2.4(1), and stabilising LTI control design procedures $K$ satisfy Assumption 2.4(2).

Although the nonlinear case is not the focus of this paper, we further remark that for nonlinear plants and controllers, Assumptions 2.4(1)-(2), also hold under appropriate Lipschitz and stabilizability assumptions; and that we expect that these conditions may be relaxed further for a nonlinear analysis without linear growth requirements. However note that the practical realisation of computationally tractable estimation schemes requires further convexity assumptions on the nonlinear plant (see Section $2 D$ ).

\section{F. The switching algorithm}

Before we explicitly define the switching algorithm we introduce the "plant generating" operator $G$. This operator is used to restrict the number of plants under consideration by the switching algorithm at any time $k \in \mathbb{N}$, and is motivated by the construction in [2] of an uncertainty for which an overly large (time-invariant) candidate plant set leads to a high closed loop gain; on the other hand a timevarying candidate plant set as specified by a suitable $G$ led to improved closed loop gain properties. We will return to this construction in Section $3 B$.

Let the "candidate" plant set under consideration be given by

$$
\mathcal{P}_{i}:=\left\{p_{1}, p_{2}, \ldots, p_{l_{i}}\right\}, p_{j} \in \mathcal{P}, 1 \leq j \leq l_{i}, i \in \mathbb{N}
$$

where

$$
\emptyset \neq \mathcal{P}_{1} \subseteq \mathcal{P}_{2} \subseteq \cdots \subseteq \mathcal{P}, \quad \cup_{i \in \mathbb{N}} \mathcal{P}_{i}=\mathcal{P}^{*} \subset \mathcal{P} .
$$

Let $\Omega=\left\{\mathcal{P}_{i} \mid i \in \mathbb{N}\right\}$. Define

$$
\begin{gathered}
G: \mathcal{W}_{e} \rightarrow \operatorname{map}(\mathbb{N}, \Omega) \\
\mathcal{P}_{i(k)}=G\left(w_{2}\right)(k), k \in \mathbb{N}
\end{gathered}
$$

subject to the constraint

$$
G\left(w_{2}\right)(0)=\mathcal{P}_{1}, G\left(w_{2}\right)(k) \subseteq G\left(w_{2}\right)(k+1), \forall k \in \mathbb{N}
$$

where $G$ defines the (time varying) plant set at time $k \in \mathbb{N}$ based on the observation $w_{2}$.
Let the minimisation operator

$$
M:\left(\operatorname{map}\left(\mathbb{N}, \operatorname{map}\left(\mathcal{P}, \mathbb{R}^{+}\right)\right), \operatorname{map}(\mathbb{N}, \Omega)\right) \rightarrow \operatorname{map}(\mathbb{N}, \mathcal{P})
$$

be given by

$$
\left[k \mapsto\left(p \mapsto r_{p}[k]\right),\left(k \mapsto \mathcal{P}_{i(k)}\right)\right] \mapsto\left[k \mapsto q_{f}(k)\right]
$$

where

$$
q_{f}(k)=\underset{p \in \mathcal{P}_{i(k)}}{\operatorname{argmin}} r_{p}[k],
$$

and we assume that $\operatorname{argmin}_{p \in \mathcal{P}_{i(k)}}, i(k) \in \mathbb{N}$ returns the parameter $p_{j}$ corresponding to the smallest index $j \in \mathbb{N}$ if there exist multiple minimal $r_{p}[k], p \in \mathcal{P}_{i}$. Hence $q_{f}(k)$ represents the plant which the estimator is determining to be the best candidate at time $k \in \mathbb{N}$. Since we want to utilise this sequence later for controller selection at time $k$, and since overly fast switching even between stabilising controllers can lead to instability [9], the EMMSAC algorithm does not switch between controllers determined by $q_{f}$, but rather by a 'slowed' version of $q_{f}$, denoted by $q \in \operatorname{map}(\mathbb{N}, \mathcal{P})$ which we introduce next.

Given a 'transition delay' function $\Delta: \mathcal{P} \rightarrow \mathbb{N}$ define the delay operator

$$
D: \operatorname{map}(\mathbb{N}, \mathcal{P}) \rightarrow \operatorname{map}(\mathbb{N}, \mathcal{P})
$$

by

$$
\left[k \mapsto q_{f}(k)\right] \mapsto[k \mapsto q(k)]
$$

where

$$
q(k)=\left\{\begin{array}{ccc}
q_{f}(k) & \text { if } \quad k-k_{s}(k) \geq \Delta\left(q\left(k_{s}(k)\right)\right) \\
q\left(k_{s}(k)\right) & \text { else }
\end{array}\right.
$$

and where $k_{s}: \mathbb{N} \rightarrow \mathbb{N}$ is given by:

$$
k_{s}(k)=\underset{0 \leq i \leq k}{\operatorname{argmax}} q(i) \neq q(i-1) .
$$

The purpose of $D$ is to delay the free switching signal $q_{f}(k)$ long enough that the stabilising effect of a corresponding plant and controller pair leads to a local finite horizon closed loop gain $\alpha<1$. This local contraction is later used to show the stability of the overall algorithm.

For $x, y, c \in \mathbb{R}$ define

$$
\lfloor c\rfloor:=\max \{n \in \mathbb{Z} \mid n \leq c\} \text { and }\left(\begin{array}{l}
x \\
y
\end{array}\right):=\frac{x !}{y !(x-y) !}
$$

where $J: \mathbb{N} \rightarrow \mathbb{N}$ is defined by

$$
J(\xi)=\xi\left(\begin{array}{c}
\xi \\
\lfloor\xi / 2\rfloor
\end{array}\right) .
$$

Let

$$
\sigma=\max \{\sigma(p), \sigma(K(p))\}, p \in \mathcal{P}^{*}
$$

and let $K: \mathcal{P} \rightarrow \mathcal{C}$ and the attenuation function $l: \mathcal{P}^{*} \rightarrow$ $[0,1)$ be given. Choose the delay $\Delta$ such that

$$
J(r) \alpha^{r}(p, K(p), \Delta(p)-\sigma, \sigma) \leq l(p)<1, \forall p \in \mathcal{P}^{*}
$$

if $1 \leq r<\infty$ and

$$
\alpha(p, K(p), \Delta(p)-\sigma, \sigma) \leq l(p)<1, \forall p \in \mathcal{P}^{*}
$$


if $r=\infty$.

In practice one would choose a stabilising design procedure $K$ and some $l: \mathcal{P}^{*} \rightarrow[0,1)$ and then compute for all $p \in \mathcal{P}^{*}$ a corresponding $\Delta(p)$ such that inequality (2.27) for $l_{r}, 1 \leq$ $r<\infty$ or inequality (2.28) for $l_{\infty}$ hold, hence we note that there always exists such a $\Delta$.

$K, \Delta$ and $l$ are design choices with individual tradeoffs. For example if we choose $l(p), p \in \mathcal{P}^{*}$ to be small for a given $K(p)$, then $\Delta(p)$ must be large to meet the given inequalities hence slowing the switching rate.

Finally define the switching operator

$$
\begin{aligned}
S: \mathcal{W}_{e} & \rightarrow \operatorname{map}(\mathbb{N}, \Omega): w_{2} \mapsto q \\
S & =D M(N E, G) .
\end{aligned}
$$

and the switching controller

$$
C: \mathcal{Y}_{e} \rightarrow \mathcal{U}_{e}: y_{2} \mapsto u_{2}
$$

for all $k \in \mathbb{N}$ by

$$
u_{2}(k)=C_{K(q(k))}\left(y_{2}-\mathscr{T}_{k_{s}(k)-1} y_{2}\right)(k)
$$

where recall that $k_{s}(k)$ is the last time $i \in \mathbb{N}, i \leq k$ s.t. $q(i) \neq q(i-1)$.

Equation (2.29) therefore ensures a zero initial condition for the atomic controller $C_{K(q(k))}$ when it is switched into closed loop at time $k_{s}(k), k \in \mathbb{N}$.

\section{Stability of The Closed loop System}

Our first objective is to establish a $l_{r}, 1 \leq r \leq \infty$ bounds on the observation $w_{2} \in \mathcal{W}_{e}$ in terms of the external disturbances $w_{0} \in \mathcal{W}$ for time varying plant sets.

We define

$$
k_{*}= \begin{cases}\min \{k \in \mathbb{N} \mid & \text { if } \exists k \text { s.t. } p_{*} \in G\left(w_{2}\right)(k) \\ \left.p_{*} \in G\left(w_{2}\right)(k)\right\} & \text { if not }\end{cases}
$$

i.e. the time at which the parameter $p_{*}$, corresponding to the unknown true plant $P=P_{p_{*}}$ belongs to the time varying set of available parameters for the first time. Note that in the classical setup e.g. of [6], [4], [8], [10], [11] we have $p_{*} \in G\left(w_{2}\right)(k)=\mathcal{P}^{*}, \forall k \in \mathbb{N}$ so $k_{*}=0$.

We now come to our first intermediate result establishing gain bounds for MIMO minimal LTI systems and time varying plant sets.

Let the interval length of consistency we require from each estimator be given by

$$
\lambda=\max _{p \in \mathcal{P}^{*}}(2 \Delta(p)+\sigma) .
$$

Theorem 3.1: Let $1 \leq r \leq \infty$. Let $p_{*} \in \mathcal{P}^{*} \subset \mathcal{P}$, $P:=P_{p_{*}}$. Let $K$ satisfy Assumptions 2.4(1)-(2). Let $S=D M(N E, G)$ be given by equations (2.9)-(2.12),(2.18)(2.26). Let $l: \mathcal{P}^{*} \rightarrow[0,1)$ be a given attenuation function and suppose that $K, \Delta, l$ satisfy inequality (2.27) for $l_{r}, 1 \leq$ $r<\infty$, or inequality (2.28) for $r=\infty$. Suppose $E$ fullfills Assumptions 2.1(1)-(4) with $\lambda$ from equation (3.31). Let $C$ be given by equation (2.29). Suppose $\left(w_{0}, w_{1}, w_{2}\right) \in$
$\mathcal{W} \times \mathcal{W}_{e} \times \mathcal{W}_{e}$ satisfy the closed loop equations (2.1),(2.2) and let $k_{*}$ be given by equation (3.30). Then:

$$
\left\|\mathscr{T}_{k} w_{2}\right\|_{r} \leq \gamma\left(G\left(w_{2}\right)(k)\right)\left(\left\|\mathscr{T}_{k_{*}-1} w_{2}\right\|_{r}+\left\|w_{0}\right\|_{r}\right)
$$

where for $\mathcal{Q} \subseteq \Omega$ :

$$
\begin{aligned}
& \sigma=\max _{p_{1}, p_{2} \in \mathcal{P}^{*}} \max \left\{\sigma\left(p_{1}\right), \sigma\left(K\left(p_{2}\right)\right)\right\} \\
& \xi=\left\{\begin{array}{ccc}
r & \text { if } & 1 \leq r<\infty \\
1 & \text { if } & r=\infty
\end{array}\right. \\
& \gamma_{1}(p)=1+\sup _{\Delta(p) \leq x \leq 2 \Delta(p)} \alpha\left(p_{*}, K(p), 0, x\right) \\
& \gamma_{2}(p)=\sup _{\Delta(p) \leq x \leq 2 \Delta(p)} \beta\left(p_{*}, K(p), 0, x\right) \\
& \alpha_{O P}(\mathcal{Q})=\max _{p \in \mathcal{Q}} l(p) \\
& \beta_{O P}(\mathcal{Q})=J(\xi) \sup _{p \in \mathcal{Q} \Delta(p) \leq x \leq 2 \Delta(p)} \sup \beta^{\xi}(p, K(p), x-\sigma, \sigma) \\
& \alpha_{O S}(\mathcal{Q})=J(\xi) \sup _{p \in \mathcal{Q} \Delta(p) \leq x \leq 2 \Delta(p)} \sup ^{\xi}(p, K(p), 0, x-\sigma) \\
& \beta_{O S}(\mathcal{Q})=J(\xi) \sup _{p \in \mathcal{Q} \Delta(p) \leq x \leq 2 \Delta(p)} \sup \beta^{\xi}(p, K(p), 0, x-\sigma) \\
& \gamma_{4}(\mathcal{Q})=\left\{\begin{array}{c}
\left(\frac{\left(1+\alpha_{O S}^{1 / r}(\mathcal{Q})\right) \alpha_{O P}(\mathcal{Q})}{1-\alpha_{O P}(\mathcal{Q})}\right)^{1 / r}+\alpha_{O S}^{1 / r}(\mathcal{Q}) \\
\quad \text { if } 1 \leq r<\infty \\
\left.\max \left\{1, \alpha_{O S}(\mathcal{Q})\right)\right\} \alpha_{O P}(\mathcal{Q})+\alpha_{O S}(\mathcal{Q}) \\
\quad \text { if } r=\infty
\end{array}\right. \\
& \gamma_{5}(\mathcal{Q})=\left\{\begin{array}{c}
\left(\frac{\left(1+\alpha_{O S}^{1 / r}(\mathcal{Q}) \beta_{O P}(\mathcal{Q})\right.}{1-\alpha_{O P}(\mathcal{Q})}\right)^{1 / r} \\
\text { if } 1 \leq r<\infty \\
\left.\max \left\{1, \alpha_{O S}(\mathcal{Q})\right\}\right) \frac{\beta_{O P}(\mathcal{Q})}{1-\alpha_{O P}(\mathcal{Q})} \\
\text { if } r=\infty
\end{array}\right. \\
& \gamma_{6}(\mathcal{Q})=\left\{\begin{array}{llc}
\beta_{O S}^{1 / r}(\mathcal{Q}) & \text { if } & 1 \leq r<\infty \\
\beta_{O S}(\mathcal{Q}) & \text { if } & r=\infty
\end{array}\right. \\
& \gamma_{7}(\mathcal{Q})=1+\gamma_{4}(\mathcal{Q}) \\
& \gamma_{8}(\mathcal{Q})=2^{1 / r} \mu|\mathcal{Q}|^{1 / r}\left(\gamma_{5}(\mathcal{Q})+\gamma_{6}(\mathcal{Q})\right) \\
& \gamma(\mathcal{Q})=\gamma_{7}^{|\mathcal{Q}|}(\mathcal{Q}) \prod_{p \in \mathcal{Q}} \gamma_{1}(p) \\
& \cdot\left(1+\left(2|\mathcal{Q}| \gamma_{8}(\mathcal{Q})+\sum_{p \in \mathcal{Q}} \gamma_{2}(p)\right)\right)
\end{aligned}
$$

Proof The (lengthy) proof is omitted, see [1].

This bound from the external disturbances to the internal signals is the direct generalisation of Theorem 4.1 in [2] from the class of dead beat stabilisable SISO systems to the class of MIMO minimal LTI systems. We now give two explicit constructions for the operator $G$ to give constructive gain and gain function bounds independent of the time $k_{*} \in \mathbb{N}$.

\section{A. Gain bounds for bounded candidate plant sets}

We first give a version of the result for the classical setup where $G$ is a constant map: $G\left(w_{2}\right)(k)=\mathcal{P}^{*}, \forall k \in \mathbb{N}$ and $p_{*} \in \mathcal{P}^{*}$. Let $\gamma$ be the gain given in Theorem 3.1. It is important to remark that the proof is constructive: an explicit expression for $\gamma$ was given above. 
Theorem 3.2: Under the conditions of Theorem 3.1 with $p_{*} \in G\left(w_{2}\right)(k)=\mathcal{P}^{*}, \forall k \in \mathbb{N}$ we have:

$$
\left\|\mathscr{T}_{k} w_{2}\right\|_{r} \leq \gamma\left(\mathcal{P}^{*}\right)\left\|w_{0}\right\|_{r}
$$

Proof Since $p_{*} \in G\left(w_{2}\right)(k)=\mathcal{P}^{*}, \forall k \in \mathbb{N}$ it follows that $k^{*}=0$ hence $\left\|\mathscr{T}_{k_{*}-1} w_{2}\right\|=0$. Hence the result follows from Theorem 3.1.

For this gain bound the robustness theory of [7] applies directly, hence the given algorithm will stabilise all plants within the gap ball of size $\gamma^{-1}\left(\mathcal{P}^{*}\right)$ around the physical plant $P_{p_{*}}$.

\section{B. Gain function bounds for unbounded candidate plant sets}

In [2] the plant

$$
P_{a}: y_{1}(k+1)=a y_{1}(k)+u_{1}(k)
$$

with an unbounded parametric uncertainty, i.e. $a \in \mathbb{Z}$ was considered. It was shown that with a MMSAC design $C$ based on atomic dead-beat controllers for a candidate plant set $\mathcal{P}=\mathbb{Z}$, the gain $w_{0} \mapsto w_{2}$ is infinite for any closed loop $\left[P_{a}, C\right], a \in \mathbb{Z}$. As previously discussed, this observation motivated the introduction of a time varying $G$ in [2] and the relaxation of gain stability to gain function stability. For the special choice of $G$ given by

$$
\begin{aligned}
G: \mathcal{W}_{e} & \rightarrow \operatorname{map}(\mathbb{N}, \Omega), \\
G\left(w_{2}\right)(k) & =\mathcal{P}_{i(k)}
\end{aligned}
$$

where for $v>2$

$$
i(k)=\left\{\begin{array}{ll}
\max \{a \in \mathbb{N} \mid & \\
\left.\gamma^{v}\left(\mathcal{P}_{a}\right)-\gamma^{v}\left(\mathcal{P}_{1}\right) \leq\left\|\mathscr{T}_{k} w_{2}\right\|\right\} & \text { if } k<\infty \\
\infty & \text { if } k=\infty
\end{array}\right\}
$$

we were then able in [2] to establish a quadratic gain function bound for the choice

$$
\mathcal{P}_{i}=\{-i,-i+1, \cdots, 0, \cdots, i-1, i\} .
$$

The general result for MIMO minimal LTI systems is established next.

Theorem 3.3: Under the conditions of Theorem 3.1 with $G$ as in equations (3.33),(3.34) we have:

$$
\left\|w_{2}\right\|_{r} \leq \gamma_{\bmod }\left(\left\|w_{0}\right\|_{r}\right)
$$

where $\gamma_{\text {mod }}: \mathbb{R}_{+} \rightarrow \mathbb{R}_{+}$is with $v>2$ given by

$$
\begin{aligned}
\gamma_{\text {mod }}(c) & =\beta_{1}+\beta_{2} c+\beta_{3} c^{2} \\
\beta_{1} & =\gamma^{v+2}\left(\mathcal{P}_{N}\right)+\gamma\left(\mathcal{P}_{N}\right) \gamma^{v}\left(\mathcal{P}_{1}\right) \\
\beta_{2} & =2 \gamma^{2}\left(\mathcal{P}_{N}\right)+\gamma^{1-v}\left(\mathcal{P}_{N}\right) \gamma^{v}\left(\mathcal{P}_{1}\right) \\
\beta_{3} & =\gamma^{2-v}\left(\mathcal{P}_{N}\right)
\end{aligned}
$$

and $N:=\min \left\{i \geq 1 \mid p_{*} \in \mathcal{P}_{i}\right\}$.

Proof The proof follows from Theorem 3.1 similarly to the proof of [2, Theorem 4.2]. Details are omitted.

Note that the given bound does not impose finiteness of $\mathcal{P}_{i}$ and only depends on $\mathcal{P}_{N}$ where $N$ is finite for any fixed $p_{*} \in \mathcal{P}^{*}$ and is a-priori determined.

\section{CONCLUSiON}

We have established a gain analysis in $l_{p}, p \in[1, \infty]$, for a class of multiple model adaptive controllers (known as EMMSAC) and for the class of MIMO minimal LTI systems. EMMSAC differs from classical MMSAC since the switching process is determined by a result of an estimation procedure, rather than the selection on the basis of observer errors. For particular choices of the plant generating operator $G$, we established a linear gain bound and a quadratic gain function bound on the map from the external disturbances to the internal signals. The latter result holds for any nested parameter set $\left\{\mathcal{P}_{i}\right\}_{i \geq 1} \subseteq \mathcal{P}$ which allows us to deal with unbounded uncertainties in the plant. Whilst the focus of the paper is in the linear setting, generalisation of the result to nonlinear plants and controllers was discussed. We have noted that much of the presented analysis is directly applicable to classes of nonlinear plants, and that generalisations to wider classes appear promising.

\section{REFERENCES}

[1] D. Buchstaller. Robust Stability and Performance for Multiple Model Switched Adaptive Control. PhD thesis, University of Southampton, School of Electronics and Computer Science (ECS), 2008, in preparation.

[2] D. Buchstaller and M.French. Scaling of gain bounds for switched adaptive control with large uncertainties. In Proc. of the 46th IEEE Conf. on Decision and Control (CDC) in New Orleans, LA, USA, pages 915-920, 2007.

[3] S. Fekri, M. Athans, and A. Pascoal. Issues, progress and new results in robust adaptive control. Int. J. of Adaptive Contr. and Signal Processing, 20(10):519-579, 2006.

[4] T. P. Fisher-Jeffes. Multiple-Model Switching Control to Achieve Asymptotic Robust Performance. $\mathrm{PhD}$ thesis, University of Cambridge, 2003.

[5] M. French. Adaptive control and robustness in the gap metric. IEEE Transactions on Automatic Control, 53(2):461-478, 2008.

[6] M. French and S. Trenn. $l^{p}$ gain bounds for switched adaptive controllers. In Proc. of the 44th IEEE Conf. on Decision and Control $(C D C)$ and the European Control Conf. (ECC) in Seville, Spain, pages 2865-2870, 2005.

[7] T. T. Georgiou and M. C. Smith. Robustness analysis of nonlinear feedback systems: An input-output approach. IEEE Transactions on Automatic Control, 42(9):1200-1220, September 1997.

[8] J. P. Hespanha, D. Liberzon, and A. S. Morse. Overcoming the limitations of adaptive control by means of logic-based switching. System and Control Letters, 49(1):49-65, 2003.

[9] D. Liberzon. Switching in Systems and Control. Birkhauser, 2003.

[10] A. Morse. Supervisory control of families of linear set-point controllers - part 1: Exact matching. IEEE Transactions on Automatic Control, 41(10):1413-1431, October 1996.

[11] A. Morse. Supervisory control of families of linear set-point controllers - part 2: Robustness. IEEE Transactions on Automatic Control, 42(11):1500-1515, November 1997.

[12] G. Vinnicombe. Examples and counterexamples in finite $\mathcal{L}_{2}$-gain adaptive control. Leuven: Sixteenth International Symposium on Mathematical Theory of Networks and Systems (MTNS2004), 2004.

[13] J. C. Willems. Deterministic least squares filtering. Journal of Econometrics, 118:341-370, 2004. 